\title{
Lumped Parametric Model for an Electrostatic Induction Micromotor Using GA
}

\author{
F. J. Santana ${ }^{\#}$, J. M. Monzón ${ }^{\#}$, S. García-Alonso*, J. A. Montiel-Nelson* \\ \# Electrical Engineering Department, University of Las Palmas de Gran Canaria \\ * Electronic and Automatic Engineering Department, University of Las Palmas de Gran Canaria \\ Campus de Tafira s/n, 35017 Las Palmas de Gran Canaria, Spain \\ E-mail: fsantana@die.ulpgc.es,jmonzon@die.ulpgc.es, sgarcia@diea.ulpgc.es,montiel@iuma.ulpgc.es
}

\begin{abstract}
In this work we introduce a novel lumped parametric equivalent circuit, that couples electromechanical parameters, and describes the behavioral model of a linear electrostatic induction micromotor. We use Genetic Algorithms (GA) for tuning the parameters of the proposed model. Our model is validated by comparison against analytical solution. The comparison results demonstrate that the fitting error between our proposed equivalent circuit and the analytical solution - calculated applying the field analytical Maxwell's equationsfor the interface potential and force density vs. slip functions are neglected.
\end{abstract}

\section{INTRODUCTION}

Researchers on electromagnetic induction machines have established some well-known models, as the classic equivalent circuit model in stationary state, that are currently published in the scientific literature [1]. Inspired by these models (IEEE Std. 112) [2] and demonstrated the duality of the electrostatic induction micromachine in the microscale with the electromagnetic induction machine in the microscale [3], a new lumped parametric equivalent circuit model is proposed for the micromotor, as shown in Fig. 1.

The quality of this circuit model is based on how its magnitudes of interest match the main phenomena under study in the micromotor (force density, tension in the interface, etc.).

The proposed lumped parametric model defines a micromotor in terms of circuit entities $\left(C_{1}, C_{2}, G_{2}\right.$, etc) and a network or topological structure. Each entity has its own constitutive equations that can be expressed in a mathematical form. The network or topological structure defines the system configuration. The composition of these mathematical relations for each entity gives us a system of algebraic equations.

The top section of Fig. 1 represents the micromotor physical model, and the bottom one shows the proposed lumped parametric equivalent circuit model. As it can be seen, the correspondence between the physical model and the lumped parametric equivalent circuit model is straightforward.

The proposed model explains and predicts the behavior of the electrostatic induction micromotor in a clear way, without expensive temporal simulations, saving computational resources. To our knowledge, no lumped parametric equivalent circuit model per phase has been found in the literature for the electrostatic induction micromotor.

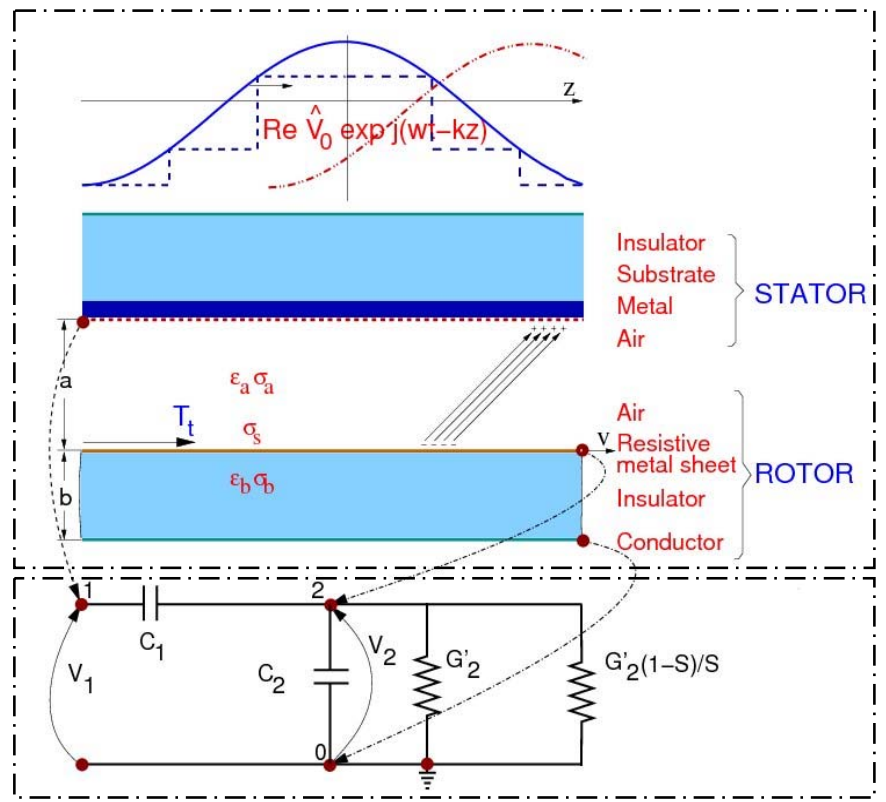

Fig. 1. Physical model and proposed per face lumped parametric equivalent models for the electrostatic induction micromotor.

\section{GENETIC ALgORITHMS}

Genetic Algorithm is an optimization technique inspired in the Darwin's evolution theory that has raised a great interest in the scientific community all over the world in recent years. This technique imitates the selection mechanics of the nature where only the more capable individuals of a population survive. John Holland was the pioneer in this topic and his main contribution was to develop the foundations that allowed the incorporation of these techniques to a computer [4].

A more precise definition of GA is [5], mathematic probabilistic searching algorithm, highly parallel, that transforms a set (population) of individual mathematical objects (associating each one with an aptitude) in a new population. That is to say, it obtains the following generation using Darwin's principles of reproduction and survival of the most capable. Between these natural genetic operations stands out the sexual recombination, the asexual recombination and the mutation. 
GA uses an analogy of the behavior of the nature, and works with a population of individuals where each one represents a possible solution of the problem. For each individual is associated a value related to its goodness that indicates the level of efficiency for competition. The greater the adaptation of an individual is, the higher is the probability of this individual to be selected for reproduction. Hence, the probability of crossing genetic material with other selected individual will be greater and the probability of propagating its genetic material to the following generation increases. If the adaptation of an individual is low, its genetic material will disappear.

From crossing genetic material, new individuals will be born defining a new generation where the offsprings share some characteristics of their parents. In this way, a new population of possible solutions replaces the previous one. An interesting property is verified, each new population will have a greater proportion of good characteristics than the previous one. Favoring the cross of the individual more adapted we explore the most interesting areas of the searching space. If the GA has been designed properly, the population will converge towards an optimal solution as the Scheme Theorem demonstrates [4]. The convergence is related with the progression towards the uniformity. A gene has converged when $95 \%$ of the individuals shear it, and the population converges when all the genes have converged.

The differences between the traditional methods and the GA methods are,

- They work with the codification of the set of parameters, not with the parameters.

- They work from a population of points, they do not start from a single point.

- They use the information of one or more objective functions, but they do not use derivates or other additional knowledge.

- They use operators and probabilistic transition rules, not deterministic rules.

- They are less affected by local maximums than the traditional techniques.

- They do not need specific knowledge about the problem they try to solve.

A few recommendations in order to achieve de convergence of the method are the following:

a) The searching space of solutions should be defined in a certain limited range, or the number of solutions should be finite.

b) It should be possible to define an objective function that indicates the goodness of a particular solution. This function controls the reproduction process.

In the work presented in this paper we have verified that these conditions are fulfilled and we have chosen genetic algorithms as a tool to tune the parameters of the proposed model.

\section{LUMPED PARAMETRIC EQUIVALENT CIRCUIT MODEL}

In this lumped parametric model for an electrostatic induction micromotor, there are not inductive impedances because they are neglected for second order effects. Instead, we can see capacitive impedances that are characteristic of the physical nature of the micromotor. The correspondence between the circuit and the physical model is based on next items:

a) The ground of the electric circuit (see point 0 in Fig. 1) coincides with the ground terminal of the electric supply applied to the micromotor.

b) The input applied voltage in the active electric circuit terminal (see point 1 in Fig. 1) coincides with the terminal of one of the six phases of the electric supply applied to the micromotor.

c) The output terminal voltage of the electrical circuit (see point 2 in Fig. 1) coincides with the voltage in the interface of the micromotor, which is the potential of the rotor. The interface is the surface of the resistive metal sheet of the rotor that is in contact with the air.

Then, we are going to compare the equivalent circuit model proposed for the induction electrostatic micromotor with the equivalent circuit model of the electromagnetic induction motor in steady state condition and referred to the stator.

In the micromotor, the electrical resistance of the stator does not exist, and the inductive impedance is replaced by its dual capacitive impedance, which is $C_{1}$ in Fig. 1.

The resistance and the inductive impedance of the rotor referred to the stator, are replaced for their duals, a conductance $G^{\prime}$ and a capacitive impedance represented for the capacitor $C_{2}$, where

$$
G_{r}^{\prime}=G_{2}^{\prime}+G_{2}^{\prime}\left(\frac{1}{S}-1\right)
$$

where the $\operatorname{slip} S$ is

$$
S=\frac{\omega / k-v}{\omega / k}
$$

and $\omega / k$ is the wave speed and $v$ the velocity of the rotor.

The relation between the lumped parametric equivalent circuit model and the physical nature of components of the micromotor is summarized as follows:

a) The capacitor $C_{1}$, placed in Fig. 1 between points 1 and 2 of the circuit, is the capacitance between two parallel plates separated by a distance, with air as dielectric material. These two planes contain the fixed and mobile elements of the electrostatic induction micromotor.

b) The capacitor $C_{2}$, placed in Fig. 1 between points 0 and 2 of the circuit, is the capacitance that exists between the superior and inferior sheets of the mobile element of the electrostatic induction micromotor. Between these sheets there is a dielectric material.

c) The conductances $G_{2}^{\prime}$ and $G_{2}^{\prime}(1-S) / S$ are, respectively, the resistance losses due to the metallic resistive sheets of the rotor, and the mechanic power generated by the micromotor which is a function of $S$. 


\section{FitTing OF THE LUMPED PARAMETERS USING GA}

As an optimization method to calculate the value of the lumped parameters of the equivalent circuit we use Genetic Algorithm techniques [6], [7]. Both the general convergence constraints and the functional parameters - population size, crossover and mutation probability, number of generations and number of pairs for generation - of the GA are defined as follows.

\section{A. Objective function}

The objective function, $\Psi$, consists in the minimization of the quadratic error between the potential in the interface, $V_{2}$, calculated in the proposed lumped parametric equivalent circuit model, and the potential calculated in the physical model of the induction electrostatic micromotor, $\Phi^{b}(\omega)$, that is to say,

$$
\Psi=\sum\left(V_{2}\left(\omega, C_{1}, C_{2}, G_{2}^{\prime}\right)-\Phi^{b}(\omega)\right)^{2}
$$

We calculate the potential in the interface in the equivalent circuit model, $V_{2}$, by a conventional method of Circuit Theory, obtaining the following equations,

$$
\begin{gathered}
Z_{1 e}=-\frac{\frac{S}{C_{2} G_{2}^{\prime} \omega} j}{\frac{S}{G_{2}^{\prime}}-\frac{1}{C_{2} \omega} j} \\
Z_{2 e}=Z_{1 e}-\frac{1}{C_{1} \omega} j \\
Z_{e d}=Z_{1 e} / Z_{2 e} \\
V_{2}=\left|\left(V_{0} Z_{e d}\right)\right|
\end{gathered}
$$

In this way, $V_{2}$ is a function of $C_{1}, C_{2}, G_{2}^{\prime}$ and $\omega$. The potential at the interface of the physical model, $\Phi^{b}$, is obtained by the following expression [3]:

$$
\Phi^{b}=\frac{V_{0}}{\sinh (k a)} \frac{\frac{\sigma_{a}}{\sigma_{e f f}}+\frac{\varepsilon_{a}}{\varepsilon_{e f f}} \omega S j}{\left(1+\frac{\varepsilon_{e f f}}{\sigma_{e f f}} \omega S j\right)}
$$

where,

$$
\begin{gathered}
\sigma_{e f f}=\sigma_{a} \operatorname{coth}(k a)+\sigma_{b} \operatorname{coth}(k b)+\sigma_{S} k \\
\varepsilon_{e f f}=\varepsilon_{a} k \operatorname{coth}(k a)+\varepsilon_{b} k \operatorname{coth}(k b)
\end{gathered}
$$

Please, note that parameters and variables are introduced in Table I.

In this paper, we present parameter values for the proposed model using $S=1$, that is to say, the slip is equal to one and so, the rotor is stopped [8]. Because, inductive impedances are neglected at the microscale [3] for another slip conditions

\begin{tabular}{|c|c|c|}
\hline Symbol & Name & Units \\
\hline$a$ & Height of dielectric $a$, air & $\mathrm{m}$ \\
\hline$b$ & Height of dielectric $b$, rotor & $\mathrm{m}$ \\
\hline$C_{l}$ & Capacitance 1 (Fig. 1) & $\mathrm{F} / \mathrm{m}$ \\
\hline$C_{2}$ & Capacitance 2 (Fig. 1) & $\mathrm{F} / \mathrm{m}$ \\
\hline$G_{2}^{\prime}$ & Conductance 2 (Fig. 1) & $\mathrm{S} / \mathrm{m}$ \\
\hline$G_{r}^{\prime}$ & Variable Conductance (Fig. 1) & $\mathrm{S} / \mathrm{m}$ \\
\hline$k$ & Number of waves per metre & - \\
\hline$j$ & Imaginary unity & - \\
\hline$S$ & Slip & - \\
\hline$T_{t}$ & Force density tangential component & $\mathrm{N} / \mathrm{m}^{2}$ \\
\hline$v$ & Linear speed of mobile part & $\mathrm{m} / \mathrm{s}$ \\
\hline$V$ & Interelectrodic voltage & $\mathrm{V}$ \\
\hline$V_{0}$ & Supply voltage & $\mathrm{V}$ \\
\hline$V_{1}$ & Voltage in point 1 (Fig. 1) & $\mathrm{V}$ \\
\hline$V_{2}$ & Voltage in point 2 (Fig. 1) & $\mathrm{V}$ \\
\hline$\varepsilon_{a}$ & Permittivity of the dielectric $a$ & $\mathrm{~F} / \mathrm{m}$ \\
\hline$\varepsilon_{b}$ & Permittivity of the dielectric $b$ & $\mathrm{~F} / \mathrm{m}$ \\
\hline$\varepsilon_{\text {eff }}$ & Effective permittivity & $\mathrm{F} / \mathrm{m}$ \\
\hline$\omega$ & Angular frequency of the signal & $\mathrm{Hz}$ \\
\hline$\sigma_{a}$ & Conductivity of the dielectric $a$ & $\mathrm{~S} / \mathrm{m}$ \\
\hline$\sigma_{b}$ & Conductivity of the dielectric $b$ & $\mathrm{~S} / \mathrm{m}$ \\
\hline$\sigma_{\text {eff }}$ & Effective Conductivity & $\mathrm{S} / \mathrm{m}$ \\
\hline$\sigma_{S}$ & Surface Conductivity & $\mathrm{S}$ \\
\hline$\Phi^{b}$ & Voltage in the interface & $\mathrm{V}$ \\
\hline$\Psi$ & GA's objective function & - \\
\hline
\end{tabular}
$\mathrm{S} \in[0,1]$ another values of the parameters are obtained following the same procedure. This implies that in the proposed equivalent circuit model, the conductance given by $G_{2}^{\prime}(1-S) / S$ remains in open circuit (the mechanic power is equal to zero because the mobile part is stopped).

TABLE I

SYMBOLS

Once we have calculated the potential $\Phi^{b}$ as a function of these variables (see equations (8) - (10)), we perform a frequency sweep, and we minimize the objective function $\Psi$ with GA, see equation (3). Based on this equation, we obtain the parameters of the equivalent circuit model, $C_{1}, C_{2}$ and $G_{2}^{\prime}$, that models the electric induction micromotor.

\section{B. GA's parameters}

We have applied Genetic Algorithms for the calculation of the particular micromotor which is represented in Fig.1. Both physic and geometric parameters are shown in Tables I and II, respectively.

A toolbox of Scilab (INRIA and ENPC retain the property rights of this open source software) has been used as a platform of numerical calculation. The problem has been solved for four values of the surface conductivity $\sigma_{\mathrm{S}}$.

We have fitted the lumped parameters, $C_{1}, C_{2}$ and $G_{2}^{\prime}$, for four different conductivities and we have observed that the capacitances $C_{1}, C_{2}$ do not vary with the conductivity, they remain constant. On the contrary, the values of the conductances are conductivity dependent. We have obtained the same conclusions with other conductivities. 
The convergence of the applied Genetic Algorithm, used for a surface conductivity $\sigma_{\mathrm{S}}=1 /\left(1800 \cdot 10^{6}\right)(1 / \Omega)$, is illustrated in Fig. 2. This plot shows the maximum and minimum values of the objective function corresponding to the 400 individuals of each generation. In $\mathrm{x}$ axis the number of generations is represented, and in y axis, in logarithmic scale, the objective function is represented. We notice that for the first generations, the error is significant, about $10^{2}$. But, as the number of generations increases, the GA improves the results, and makes that both error values converge and tend to zero. It can be seen that 40 generation onwards, the objective function is lower than about $10^{-3}$, as illustrated in Fig. 2 .

Table III shows the parameters that have been used for the GA calculation. These values have been used in all simulations and for each surface conductivity of the material. The computation time was always less than two minutes for a single process.

TABLE II

PhysicAl AND GeOMETRICAL PARAMETERS OF THE MicromaChINE

\begin{tabular}{|c|c|r|c|}
\hline Symbol & Name & \multicolumn{1}{c|}{ Value } & Unit \\
\hline$L$ & Length of the structure & $44 \cdot 10^{-6}$ & $\mathrm{~m}$ \\
\hline$h m$ & Height of the metallic plates & $0.01 \cdot 10^{-6}$ & $\mathrm{~m}$ \\
\hline$a$ & Height of dielectric 2 & $3 \cdot 10^{-6}$ & $\mathrm{~m}$ \\
\hline$b$ & Height of dielectric 1 & $10 \cdot 10^{-6}$ & $\mathrm{~m}$ \\
\hline$k$ & Number of waves per metre & $2 \pi / \mathrm{L}$ & $\mathrm{m}^{-1}$ \\
\hline$v$ & Linear speed of mobile part & 0 & $\mathrm{~m} / \mathrm{s}$ \\
\hline$f$ & Temporal frequency of excitation & $2.6 \cdot 10^{6}$ & $\mathrm{~Hz}$ \\
\hline$V_{0}$ & Maximum value of excitation & 200 & $\mathrm{~V}$ \\
\hline
\end{tabular}

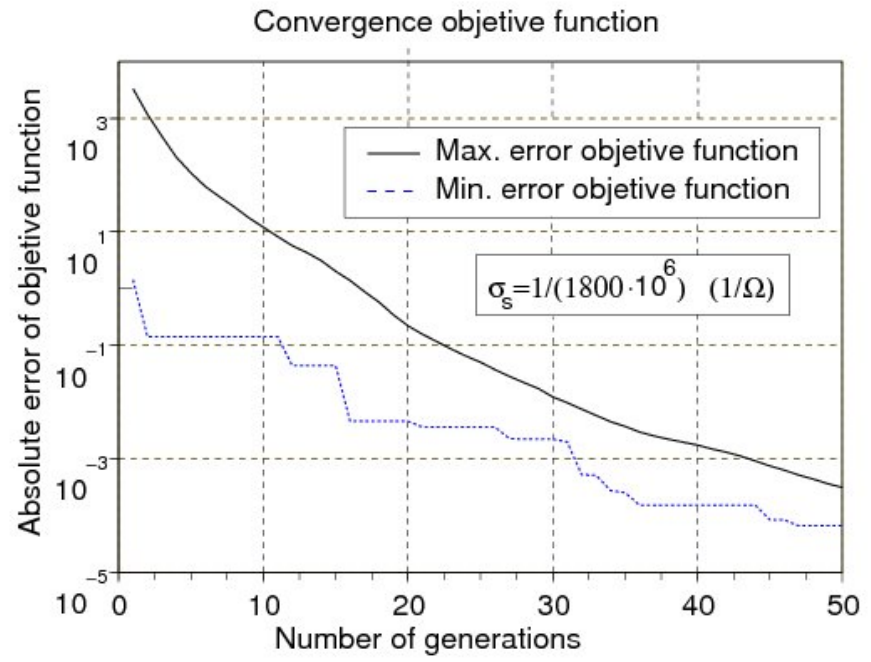

Fig. 2. Absolute error of objective function.

TABLE III

GA'S PARAMETERS

\begin{tabular}{|l|r|}
\hline Name & Value \\
\hline Population size & 400 \\
\hline Crossover probability & 0.7 \\
\hline Mutation probability & 0.05 \\
\hline Number of generations & 50 \\
\hline Number of pairs & 150 \\
\hline
\end{tabular}

\section{RESUlTS AND MOdEL VALIDATION}

Table IV contains the optimized values for the parameters of the lumped model per phase and unit of width. Note that when the material conductivity of the metallic sheet of the rotor increases, the values of the capacities $C_{1}$ and $C_{2}$ remain approximately invariable. However, the conductivity $G_{2}^{\prime}$ increases.

TABLE IV

CAlculated VAlues for LuMPed PARAMETERS PER PHASE

\begin{tabular}{|c|c|c|c|}
\hline $\begin{array}{c}\sigma_{S} \\
(1 / \Omega)\end{array}$ & $\begin{array}{c}\mathrm{C}_{1} \\
(\mathrm{~F} / \mathrm{m})\end{array}$ & $\begin{array}{c}\mathrm{C}_{2} \\
(\mathrm{~F} / \mathrm{m})\end{array}$ & $\begin{array}{c}\mathrm{G}_{2}^{\prime} \\
\left(\Omega^{-1} / \mathrm{m}\right)\end{array}$ \\
\hline $1 /\left(1800 \cdot 10^{6}\right)$ & $238.71857 \cdot 10^{-9}$ & $483.966 \cdot 10^{-9}$ & $9.4506 \cdot 10^{-1}$ \\
\hline $1 /\left(600 \cdot 10^{6}\right)$ & $238.58074 \cdot 10^{-9}$ & $483.283 \cdot 10^{-9}$ & $28.3215 \cdot 10^{-1}$ \\
\hline $1 /\left(200 \cdot 10^{6}\right)$ & $238.58724 \cdot 10^{-9}$ & $483.306 \cdot 10^{-9}$ & $84.9662 \cdot 10^{-1}$ \\
\hline $1 /\left(144 \cdot 10^{6}\right)$ & $238.58507 \cdot 10^{-9}$ & $483.294 \cdot 10^{-9}$ & $118.007 \cdot 10^{-1}$ \\
\hline
\end{tabular}

From the results we demonstrate that the relation between the surface conductivity $\left(\sigma_{S}\right)$ of the physic model and the conductance $\left(G_{2}^{\prime}\right)$ of the lumped parametric equivalent circuit model is linear, as illustrated in Fig. 3.

Figure 4 shows the potential at the interface versus slip for the physical model. This was calculated through analytical equations obtained through the field Maxwell's equations [3], and for the equivalent lumped circuit model, calculated through GA. Both curves are coincident, so the model has been validated. The mean square error for 50 generations is lower than $0.0000015 \%$.

Figure 5 illustrates the relationship between force density and slip for the physical model. We obtained it by solving both analytical equations [3], [8] and the proposed equivalent circuits. Both curves are coincident and the mean square error for 50 generations is lower than $0.0000006 \%$. Once again, the proposed equivalent circuit is validated.

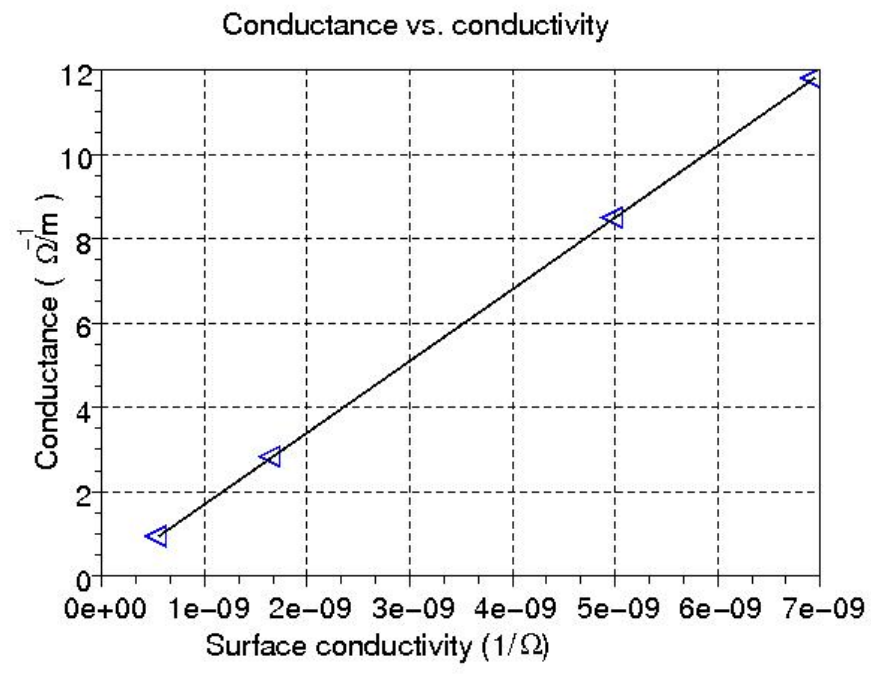

Fig. 3. Linear relation detected between superficial conductivity and conductance. 


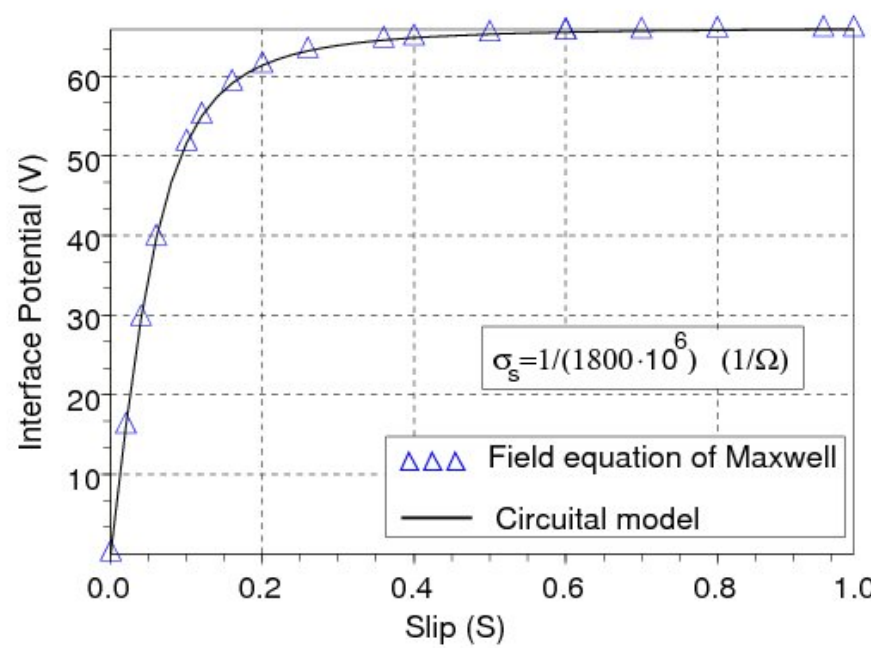

Fig. 4. Model validation: Potential in the interface vs. slip obtained both by the circuital model and from the field equation of Maxwell.

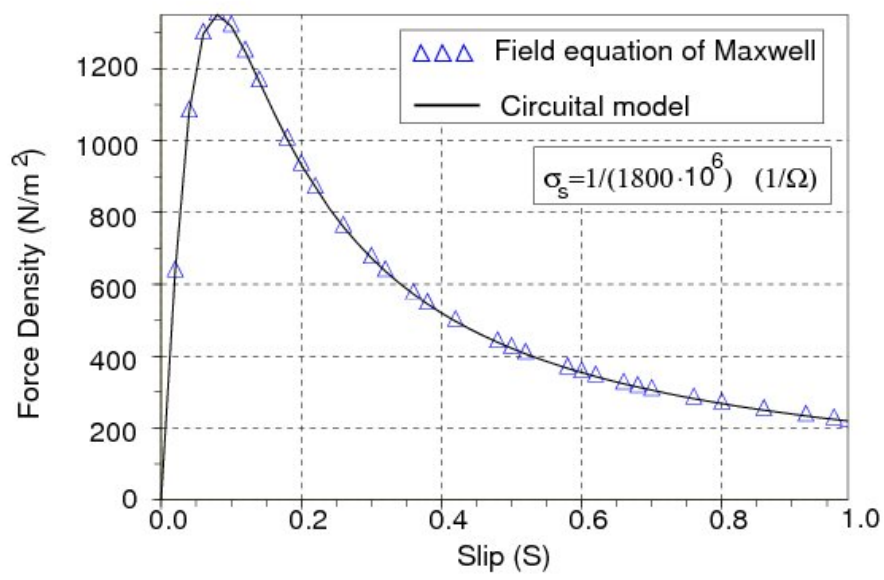

Fig. 5. Model validation: Force density vs. slip obtained both by the circuital model and from the field equation of Maxwell.

\section{CONCLUSIONS}

We have introduced a novel lumped parametric equivalent circuit model per phase in stationary state that couples electromechanical parameters, describing the behavioral model of a linear electrostatic induction micromotor. We have used Genetic Algorithms for tuning the parameters, of the proposed model. Our model has been validated by comparison against analytical solution. The comparison results demonstrate that the fitting error between our proposed equivalent circuit and the analytical solution - calculated applying the field analytical Maxwell's equations - for the interface potential and force density vs. slip functions are neglected.

\section{ACKNOWLEDGMENT}

The authors gratefully acknowledge the collaboration of the MEMS Division of IUMA (Institute for Applied Microelectronics) at the University of Las Palmas de Gran Canaria, Spain.

\section{REFERENCES}

[1] Saffet Ayasun and Chika O. Nwankpa, "Induction Motor Test Using Matlab/Simulink and Their Integration Into Undergraduate Electric Machinery Courses", IEEE Trans. On Education., vol. 48, no. 1, Feb, 2005.G.

[2] IEEE Standard Test Procedure for Polyphase Induction Motors and Generators, IEEE Standard 112-2004, Nov. 2004.

[3] F. J. Santana, J. M. Monzón, S. García-Alonso, J. A. Montiel-Nelson, "Analysis and Modeling of an Electrostatic Induction Micromotor", in Proc. of the IEEE ICEM-08, Villamoura, Portugal, Sept. 2008.

[4] J. H. Holland, "Adaptation in Natural and Artificial Systems", MIT Press, New York, 1975.

[5] J. R. Koza, "Genetic Programming: On the Programming of Computers by Means of Natural Selection", MIT Press ISBN 026211170 5, 1992.

[6] B. Abdelhadi, A. Benoudjit, N. Nait-Said, "Application of Genetic Algorithm with a Novel Adaptive Scheme for the Identification of Induction Machine Parameters", IEEE Trans. On Energy Conversion., vol. 20, no. 2, June, 2005.

[7] G. Leu, S. Simon, A. Serbanescu, "MEMS optimization using genetic algorithm" 2004 International Semiconductor Conference, vol. 2, pp. 475-478. 2005

[8] F. J. Santana, J. M. Monzón, S. García-Alonso, J. A. Montiel-Nelson, "Operation Modes of an Electrostatic Induction Micromachine", DCIS08, in Proc. of DCIS-08, Grenoble, France, Nov. 2008. 